\title{
Renewable synthesis of $n$-butyraldehyde from glucose by engineered Escherichia coli
} CrossMark

\author{
Jason T. Ku ${ }^{2}$ Wiwik Simanjuntak ${ }^{1}$ and Ethan I. Lan ${ }^{1 *}$ [D
}

\begin{abstract}
Background: $n$-Butyraldehyde is a high-production volume chemical produced exclusively from hydroformylation of propylene. It is a versatile chemical used in the synthesis of diverse C4-C8 alcohols, carboxylic acids, esters, and amines. Its high demand and broad applications make it an ideal chemical to be produced from biomass.

Results: An Escherichia coli strain was engineered to produce $n$-butyraldehyde directly from glucose by expressing a modified Clostridium CoA-dependent $n$-butanol production pathway with mono-functional Coenzyme A-acylating aldehyde dehydrogenase (Aldh) instead of the natural bifunctional aldehyde/alcohol dehydrogenase. Aldh from Clostridium beijerinckii outperformed the other tested homologues. However, the presence of native alcohol dehydrogenase led to spontaneous conversion of $n$-butyraldehyde to $n$-butanol. This problem was addressed by knocking out native E. coli alcohol dehydrogenases, significantly improving the butyraldehyde-to-butanol ratio. This ratio was further increased reducing media complexity from Terrific broth to M9 media containing 2\% yeast extract. To increase production titer, in situ liquid-liquid extraction using dodecane and oleyl alcohol was investigated. Results showed oleyl alcohol as a better extractant, increasing the titer of $n$-butyraldehyde produced to $630 \mathrm{mg} / \mathrm{L}$.
\end{abstract}

Conclusion: This study demonstrated $n$-butyraldehyde production from glucose. Through sequential strain and condition optimizations, butyraldehyde-to-butanol ratio was improved significantly compared to the parent strain. Results from this work may serve as a basis for further development of renewable $n$-butyraldehyde production.

Keywords: Butanal, Butanol, CoA-acylating aldehyde dehydrogenase

\section{Background}

Commodity chemical industry relies almost entirely on non-renewable petroleum and other fossil fuel resources, leading to environmental pollutions and the inevitable depletion crisis. As a potential solution, renewable chemical production through microbial conversion of biomass is an attractive direction for sustainability. However, chemicals natural to biological production are limited in quantity and type. Therefore, synthetic metabolic pathways are designed and engineered into microorganisms for increasing both the amounts and types of chemicals accessible to bio-based conversion.

$n$-Butyraldehyde is a large volume chemical produced exclusively from hydroformylation of propylene (Fig. 1a)

\footnotetext{
${ }^{*}$ Correspondence: ethanilan@nctu.edu.tw

1 Department of Biological Science and Technology, National Chiao Tung

University, 1001 Daxue Road, Hsinchu 300, Taiwan

Full list of author information is available at the end of the article
}

with annual production of 7 million tons and estimated growth of $2-3 \%$ per year [1]. As a reactive chemical, $n$-butyraldehyde is a versatile intermediate for the synthesis of various $\mathrm{C} 4$ and $\mathrm{C} 8$ alcohols, carboxylic acids, amines, and esters. In particular, 2-ethylhexanol has been a large volume derivative from $n$-butyraldehyde for its use in synthesizing phthalate plasticizers. Furthermore, $n$-butyraldehyde is also the precursor to polyvinyl butyral, a common polymer used for laminated glass in automotive and architectural industries. Recently, $n$-butyraldehyde has also been shown to be a biological precursor for bio-propane production [2]. These widespread applications of $n$-butyraldehyde make it an ideal chemical to be produced renewably.

Biological production of aldehydes is limited due to toxicity and reactivity. While few aldehyde products have been produced by engineered microbes [3-6], the biochemical repertoire for aldehydes needs to be expanded 


\section{a Chemical Process}

\section{Applications}

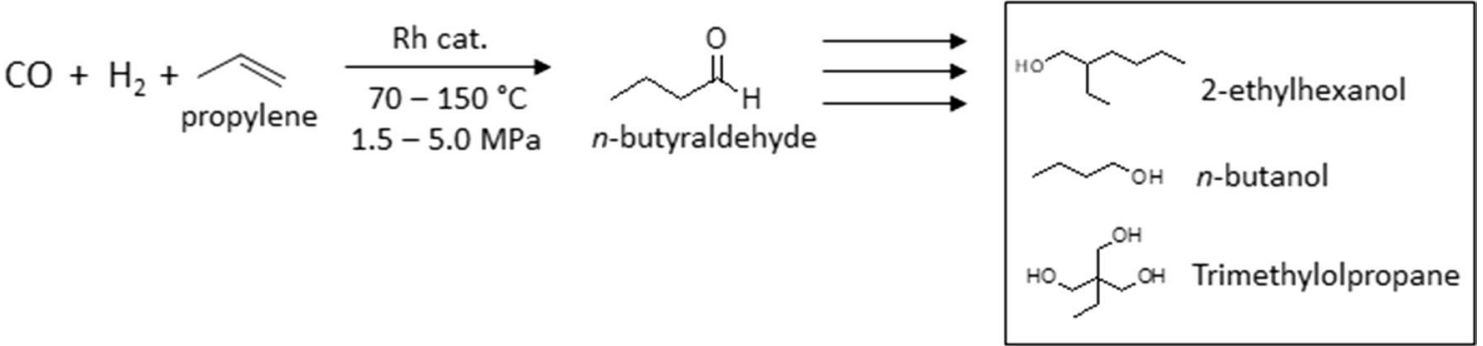

\section{b Synthetic metabolic pathway}
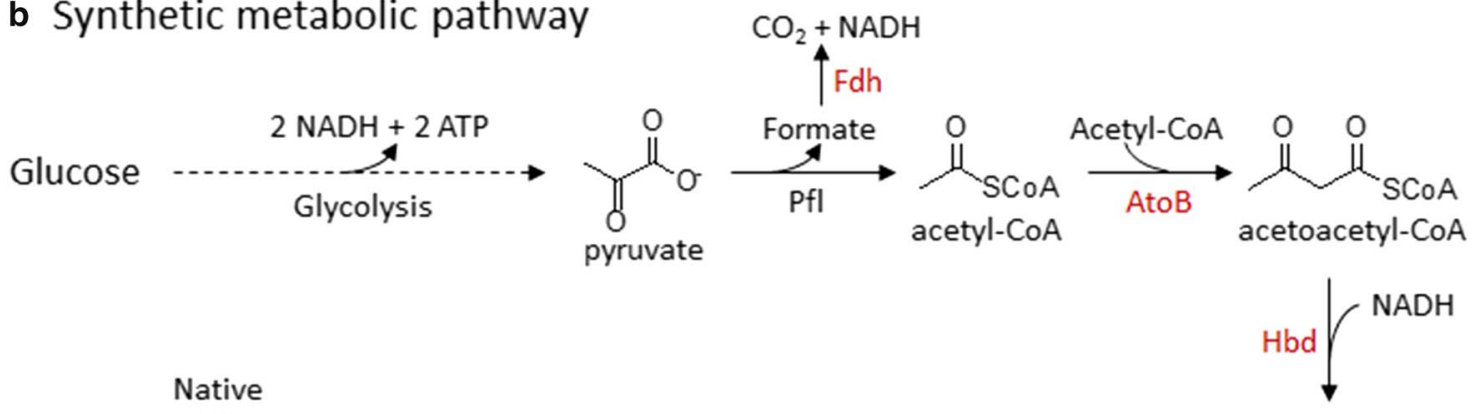

Fig. 1 a Chemical synthesis of $n$-butyraldehyde from petrochemical feedstock. Propylene and syngas are reacted under high temperature and pressure to form $n$-butyraldehyde. Commercially important downstream products are shown as representative applications. b Metabolic pathway for $n$-butyraldehyde biosynthesis from glucose. Six genes are overexpressed to produce $n$-butyraldehyde. Native adh genes coding for alcohol dehydrogenases are knocked out to prevent excessive reduction of $n$-butyraldehyde. Fdh formate dehydrogenase; AtoB acetyl-CoA acetyltransferase; Hbd 3-hydroxybutyryl-CoA dehydrogenase; Crt crotonase; Ter trans-enoyl-CoA reductase; Aldh CoA-acylating aldehyde dehydrogenase

to support the effort in sustainability. $n$-Butyraldehyde has been previously reported in a mutant strain of Clostridium acetobutylicum [7] lacking alcohol dehydrogenase, capable of secreting up to $1.6 \mathrm{~g} / \mathrm{L}$ of $n$-butyraldehyde. However, Clostridia are more difficult to work with than other well-characterized microorganism such as Escherichia coli and Saccharomyces cerevisiae due to their complex physiology and metabolism, as well as having less developed genetic manipulation tools. In addition, facultative anaerobes such as $E$. coli are often preferred for bio-based chemical productions because they grow rapidly during aerobic cultivation and conserves carbon for production under anaerobic conditions, increasing product yield due to elimination of respiration. Therefore, commercial interests are in engineering $E$. coli for $n$-butyraldehyde production [8]. However, due to the presence of numerous native alcohol dehydrogenases (Adh) in E. coli, n-butyraldehyde is spontaneously converted to $n$-butanol, thereby lowering the yield of aldehyde. This same behavior was observed in isobutyraldehyde production in E. coli [4]. Through knocking out several endogenous genes coding for Adh in E. coli, isobutyraldehyde production was significantly improved to roughly $2.5 \mathrm{~g} / \mathrm{L}$ in test tubes and up to $35 \mathrm{~g} / \mathrm{L}$ with gas stripping as in situ product removal. Inspired by the $E$. coli isobutyraldehyde production, here we also deleted adh genes and showed significant improvement in $n$-butyraldehyde production. Furthermore, in the process of constructing a $n$-butyraldehyde production pathway, we identified an alternative and better CoA-acylating aldehyde dehydrogenase than what has been previously reported. Lastly, instead of using gas stripping for in situ product removal as has been demonstrated for isobutyraldehyde, we tested in situ removal through organic overlay for liquid-liquid extraction and showed that oleyl alcohol is a suitable extractant for $n$-butyraldehyde production. The results obtained from this study provided a method for renewable synthesis of $n$-butyraldehyde with significantly reduced butanol co-production.

\section{Methods}

Strains and plasmids construction

Strains and plasmids used in this study are listed in the Additional file 1: Table S1. Primer sequences used are 
listed in Additional file 1: Table S1. Strains ELeco1 to KS8 were constructed from JCL299 [9] by sequential deletion of aldehyde reductase genes. All gene deletions were carried out using P1 transduction [10] with Keio collection [11] as donor strains. Kanamycin resistance marker was removed via FLP-mediated recombination. The successful gene deletions were subsequently verified by PCR (Additional file 1: Figure S1). All plasmids in this study were constructed using Gibson assembly [12]. Plasmids pKU48, pKU49, pKU50, and pKU51 were constructed by replacing adhE2 in pRW13 with aldh (CB), aldh (CB(mut)), aldh (CS), and aldh (CS(N1-4)), respectively. Briefly, a fragment containing plasmid vector, $a t o B$, $c r t$, and $h b d$ was amplified using primers KU115 and KU116 using pRW13 as a template. This fragment was assembled with individual aldh fragments amplified by the specified primers in Additional file 1: Table S1 using the genomic DNA of the corresponding source organism. aldh ( $\mathrm{CB}(\mathrm{mut}))$ gene was cloned from a cloning vector containing the gene in our lab collection. For its sequence, see Additional file 1. Plasmids were then verified through sequencing.

\section{Culture media and growth conditions}

All chemicals were purchased from Sigma-Aldrich or JTBaker. Media were purchased from BD-biosciences. All E. coli strains were cultured at $37{ }^{\circ} \mathrm{C}$ in a rotatory shaker (250 rpm). Luria broth (LB) and LB plates $(1.5 \% \mathrm{w} / \mathrm{v}$, agar) were routinely used for $E$. coli cultivation unless otherwise specified. Terrific broth (TB; 12 g tryptone, 24 g yeast extract, $2.31 \mathrm{~g} \mathrm{KH}_{2} \mathrm{PO}_{4}, 12.54 \mathrm{~g} \mathrm{~K}_{2} \mathrm{HPO}_{4}, 4 \mathrm{~mL}$ glycerol per liter of water) supplemented with $20 \mathrm{~g} / \mathrm{L}$ glucose was used as complex medium for $n$-butyraldehyde production. For medium analysis, M9 medium (12.8 g $\mathrm{Na}_{2} \mathrm{HPO}_{4} \cdot 7 \mathrm{H}_{2} \mathrm{O}, 3$ g K ${ }_{2} \mathrm{PO}_{4}, 0.5 \mathrm{~g} \mathrm{NaCl}, 0.5 \mathrm{~g} \mathrm{NH}_{4} \mathrm{Cl}$, $1 \mathrm{mM} \mathrm{MgSO}_{4}, 1 \mathrm{mg}$ vitamin $\mathrm{B} 1$ and $0.3 \mathrm{mM} \mathrm{CaCl}_{2}$ per liter of water) supplemented with $20 \mathrm{~g} / \mathrm{L}$ glucose and various concentrations $(0.125-2.0 \%)$ of yeast extract (YE) and tryptone were used. When required, antibiotics were added into culture medium for selection at the following concentrations: kanamycin (Kan), $50 \mu \mathrm{g} / \mathrm{mL}$; chloramphenicol (Cm), $50 \mu \mathrm{g} / \mathrm{mL}$; ampicillin (Amp), $100 \mu \mathrm{g} / \mathrm{mL}$; tetracycline (Tet), $15 \mu \mathrm{g} / \mathrm{mL}$. Cell growth was routinely determined by measuring optical density at wavelength of $600 \mathrm{~nm}\left(\mathrm{OD}_{600}\right)$ of cultures using a Biotek epoch 2 microplate spectrophotometer. Path length was adjusted to $1 \mathrm{~cm}$.

\section{n-Butyraldehyde production}

$1 \%(\mathrm{v} / \mathrm{v})$ of overnight cultures in LB was used to inoculate $3 \mathrm{~mL}$ of production media (TB or M9 with varying concentration of yeast extract) containing $20 \mathrm{~g} / \mathrm{L}$ glucose with appropriate antibiotics in test tubes. When the cultures reached $\mathrm{OD}_{600}$ of $0.4-0.6$, they were switched to anaerobic by transferring them into a $10-\mathrm{mL}$ BD vacutainer tube. Head space was then purged with anaerobic gas $\left(95 \% \mathrm{~N}_{2}, 5 \% \mathrm{H}_{2}\right)$. Cultures were then sampled at specified times for optical density measurement and product quantification.

\section{In situ removal of $n$-butyraldehyde by liquid-liquid extraction}

$1 \%(\mathrm{v} / \mathrm{v})$ of overnight cultures were inoculated into $20 \mathrm{~mL}$ TB supplemented with $20 \mathrm{~g} / \mathrm{L}$ glucose in $250-\mathrm{mL}$ baffle flasks. When the culture OD600 reached 0.4-0.6, and $10 \mathrm{~mL}$ or $20 \mathrm{~mL}$ of either dodecane or oleyl alcohol was added as extractant to the culture. Subsequently, the cultures were switched to anaerobic by placing them in BD GasPak Anaerobe Gas Generating Pouch System with Indicator. When taking samples, anaerobic pouch was opened inside an anaerobic chamber to maintain the anaerobic environment.

\section{Product quantification}

Culture samples $(1 \mathrm{~mL})$ were centrifuged at $10,000 \times g$ for $5 \mathrm{~min}$. The supernatants were then collected for product analysis. When analyzing product content in extractant, extractant was diluted using ethyl acetate. Aldehyde and alcohol concentrations in both medium and extractant were quantified by a Shimadzu GC-2010 gas chromatography (GC) equipped with a barrier ionization discharge (BID) detector. The separation of compounds was performed by SH-Rtx-wax GC column $(30 \mathrm{~m}, 0.32 \mathrm{~mm}$ i.d., $0.50-\mu \mathrm{m}$-thick film). GC oven temperature was initially held at $40{ }^{\circ} \mathrm{C}$ for $2 \mathrm{~min}$ and increased with a gradient of $5{ }^{\circ} \mathrm{C} / \mathrm{min}$ until $80{ }^{\circ} \mathrm{C}$ followed by a gradient of $12{ }^{\circ} \mathrm{C} / \mathrm{min}$ until $120{ }^{\circ} \mathrm{C}$. Then the temperature continues to rise with a gradient of $20{ }^{\circ} \mathrm{C} / \mathrm{min}$ until $230{ }^{\circ} \mathrm{C}$ and held for $2 \mathrm{~min}$. Helium was used as the carrier gas. The injector was maintained at $220{ }^{\circ} \mathrm{C}$, and the detector was maintained at $230{ }^{\circ} \mathrm{C} .1 \mu \mathrm{L}$ of samples was injected in split injection mode (1:15 split ratio) using 2-methyl1-pentanol or 1-pentanol as the internal standard. Glucose consumption was determined by subtracting the glucose concentration in samples from the concentration in original medium. Glucose concentration was measured using Agilent 1260 HPLC equipped with a refractive index detector. The injection volume used was $20 \mu \mathrm{L}$. The mobile phase consisted of $5 \mathrm{mM} \mathrm{H}_{2} \mathrm{SO}_{4}$ with a linear flow rate of $0.6 \mathrm{~mL} / \mathrm{min}$. Separation of metabolites was done by Agilent HiPlex- $\mathrm{H}(700 \times 7.7 \mathrm{~mm})$ organic acid analysis column maintained at $65^{\circ} \mathrm{C}$. A Bio-Rad Micro-Guard Cation $\mathrm{H}$ guard column $(30 \times 4.6 \mathrm{~mm})$ was connected in front of the analysis column. Glucose was monitored 
by refractive index detector. Concentration of glucose in the collected samples was determined by standard curve constructed from HPLC analysis of standard glucose solutions.

\section{Partition coefficient determination for $\boldsymbol{n}$-butyraldehyde in dodecane and oleyl alcohol}

To measure the partition coefficient of $n$-butyraldehyde for dodecane and oleyl alcohol, $800 \mu \mathrm{L}$ of different concentrations of $n$-butyraldehyde (0.01, 0.02, $0.05,0.1,0.2 \%$ in water) were mixed with the same volume organic extractant in glass GC vials. The mixtures were sealed and mixed by vortex for 1 min followed by a 36-h incubation in $37^{\circ} \mathrm{C}$. After incubation, the concentrations of $n$-butyraldehyde in each phase were determined by GC as described in "Methods" section. The partition coefficients were calculated using the equation below:

$$
\text { Partition coefficient }=\log \frac{[\text { Butyraldehyde }]_{\text {organic }}}{[\text { Butyraldehyde }]_{\text {water }}} .
$$

\section{Results and discussion}

Selection of CoA-acylating aldehyde dehydrogenase for $n$-butyraldehyde production

$n$-Butyraldehyde is an intermediate in the Clostridium CoA-dependent $n$-butanol production pathway $[9,13$, 14] (Fig. 1b). However, bifunctional aldehyde/alcohol dehydrogenase AdhE2 catalyzes the direct two-step conversion of butyryl-CoA to $n$-butanol, bypassing $n$-butyraldehyde as a product. To avoid conversion of $n$-butyraldehyde to $n$-butanol, we first replaced AdhE2 with CoA-acylating aldehyde dehydrogenase (Aldh), catalyzing only the conversion of butyryl-CoA to $n$-butyraldehyde. Some Clostridia such as Clostridium beijerinckii contain individual Aldh and Adh instead of a bifunctional enzyme for butanol production. Alternatively, Aldh is found in the degradation pathways for ethanolamine and 1,3-propanediol $[15,16]$. However, the Aldh from ethanolamine and 1,3-propanediol utilization operons are not specific for butyryl-CoA reduction and have been previously shown to produce ethanol when expressed in E. coli [17]. Therefore, for the present study, we chose to work with Clostridium Aldh. Based on the sequence of aldh from C. beijerinckii [18], we selected two additional homologues from $C$. saccharolyticum and C. saccharoperbutylacetonicum, as well as a mutant aldh from $C$. beijerinckii which we isolated previously in our lab (see Additional file 1 for its sequence). These four aldh genes were individually cloned into synthetic operons with the genes necessary to convert acetyl-CoA to butyryl-CoA (Fig. 1b). These synthetic operons were driven by native E. coli promoter of ack and $a d h E$ genes, $\mathrm{P}_{\text {ack }}$ and $\mathrm{P}_{\mathrm{adhE}}$, respectively, which have been previously shown to produce higher titers of butanol compared to using IPTGinducible $\mathrm{P}_{\text {LlacO1 }}$ promoter [19]. Here the $\mathrm{P}_{\text {ack }}$ and $\mathrm{P}_{\text {adhe }}$ were defined to include the ribosomal binding site and $5^{\prime}$ untranslated region upstream of their corresponding genes. atoB, aldh, crt, and $h b d$ were cloned as one operon on a colE1 origin plasmid under the control of $\mathrm{P}_{\text {ack. }}$. ter and $f d h$ were individually expressed on colA and pSC101 origin plasmids, respectively, under the control of $\mathrm{P}_{\text {adhe }}$ [19]. These plasmids were transformed into $E$. coli strain JCL299 which was previously shown to efficiently produce $n$-butanol and has $l d h A, a d h E, f r d B C$, and $p t a$ knocked out [9]. Having the mixed acid fermentation pathways knocked out, JCL299 efficiently channels acetyl-CoA and NADH for the synthesis of $n$-butyraldehyde. As expected, due to the presence of endogenous alcohol dehydrogenases, the resulting strains showed minimal production of $n$-butyraldehyde (Fig. 2a). Majority of the fermentation products was $n$-butanol across the strains expressing the four different aldh genes. Chromosomal $y q h D$, coding for NADPH-dependent alcohol dehydrogenase, is known to reduce aldehydes to their corresponding alcohols and highly active as a detoxification mechanism [20-22]. Therefore, we knocked out $y q h D$ in JCL299, yielding strain ELeco1. Expressing the $n$-butyraldehyde pathway in strain ELeco1, $n$-butyraldehyde production was observed. The best strain ELeco1/ pKU48/pRW18/pRW22 produced $0.16 \mathrm{~g} / \mathrm{L}$ of $n$-butyraldehyde (Fig. 2b). However, the aldehyde-to-alcohol ratio was 0.39 . This low aldehyde-to-alcohol ratio indicates the presence of other active native Adh capable of reducing $n$-butyraldehyde.

\section{Improving aldehyde-to-alcohol ratio by knocking out native alcohol dehydrogenases}

To decrease $n$-butanol formation and increase aldehydeto-alcohol ratio, we knocked out the genes coding for other native Adh. Based on previous work for isobutyraldehyde production [23], we deleted eight $a d h$ genes that are likely to contribute to $n$-butyraldehyde reduction: $y j g B$, fucO, eut $G, y b b O$, adhP, gldA, $y a h K$, and $y g h A$. These genes were selected because their knockouts led to higher production titers of isobutyraldehyde. We sequentially knocked out each of these $a d h$ genes using P1 phage transduction with the Keio collection. Since all of these adh genes have been shown to be effective for increasing isobutyraldehyde production, they were knocked out without specific order. The results of $n$-butyraldehyde production titers and the aldehyde-to-alcohol ratio from these mutant strains are shown in Fig. 3. We noted that the titers achieved by the ELeco1/pKU48/ pRW18/pRW22 are comparable to those achieved by the 

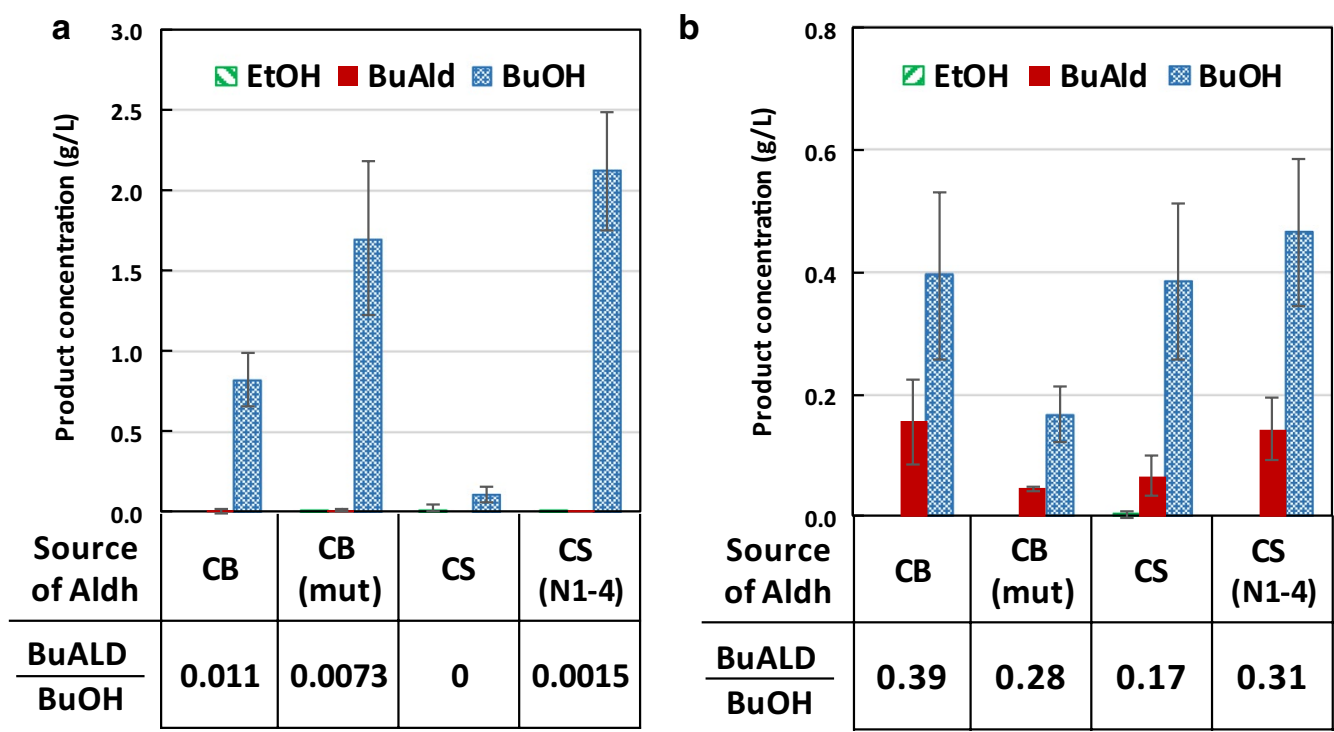

Fig. 2 Production of butyraldehyde, butanol, and their ratios by strain a JCL299 and b ELeco1 expressing different Aldh with CoA-dependent pathway. CB, C. beijerinckii; CB (mut), mutant Aldh from C. beijerinckii; CS, C. saccharobutylicum; CS (N1-4), C. saccharoperbutylacetonicum N1-4; BuALD, $n$-butyraldehyde; $\mathrm{BuOH}, n$-butanol. Error bars represent standard deviation of three experiments

published patent application from Easel Biotechnologies [8], which used a strain with identical genotype. However, because the $n$-butanol titers were not reported in their work, the butyraldehyde-to-butanol ratios cannot be compared. Nevertheless, here we took the strain design further and showed that additional knockouts of native $a d h$ genes led to significant reduction in butanol. The final strain KS8/pKU48/pRW18/pRW22 reached an aldehyde-to-alcohol ratio of 3.1, representing an eightfold improvement compared to strain ELeco1/pKU48/ pRW18/pRW22. Here we noted that strain KS7 harboring the same plasmids reached slightly higher aldehyde-toalcohol ratio of 3.4. However, the difference in aldehydeto-alcohol ratios was within error range and insignificant. Therefore, strain KS8 was used for downstream experiments. Among the eight additional $a d h$ that we knocked out, eut $G, y b b O$, and $y g h A$ showed no effect for increasing aldehyde-to-alcohol ratio. All other adh knock out contributed positively towards increasing aldehydeto-alcohol ratio, indicating their native expression and corresponding enzymes' capability of reducing $n$-butyraldehyde. While aldehyde-to-alcohol ratio increased with each $a d h$ gene knock out, the titer of $n$-butyraldehyde did not significantly increase. Correspondingly, glucose consumption by strains with each additional $a d h$ gene knock out also decreased (Fig. 3). These results indicate that carbon flux was reduced. Analysis of the thermodynamics of each step revealed that butyryl-CoA reduction to $n$-butyraldehyde is thermodynamically unfavorable with
$\Delta \mathrm{G}^{\circ \circ}$ of $7.7 \mathrm{~kJ} / \mathrm{mol}$ (calculated using eQuilibrator [24]), which may lead to inefficient conversion of butyryl-CoA to $n$-butyraldehyde, particularly after $n$-butyraldehyde concentration reached a certain threshold. It is likely that as $n$-butyraldehyde production is slowed, glucose metabolism also slows due to inability for NADH recycling. Since the native $E$. coli fermentation genes ( $a d h E$, frdBC, and $l d h A$ ) have been knocked out in strain KS8, $n$-butyraldehyde production becomes the only fermentative pathway available to recycle NADH back to $\mathrm{NAD}^{+}$. When $n$-butyraldehyde biosynthesis is slowed down, less $\mathrm{NAD}^{+}$is available for use in glycolysis. As a result, glucose consumption rate decreases. Therefore, we next investigated the effect of in situ removal on $n$-butyraldehyde production titer.

\section{Improving $n$-butyraldehyde titer by in situ product removal}

Here we chose to use in situ liquid-liquid extraction for $n$-butyraldehyde removal using organic overlay. Dodecane and oleyl alcohol were selected as extractants because of their general application and non-toxicity to microbial cultures $[25,26]$. We determined the partition coefficient of $n$-butyraldehyde in water and the two organic solvents by measuring the ratio of $n$-butyraldehyde appearing in both aqueous and organic phase (Additional file 1: Figure S2) after vigorous mixing followed by stationary incubation at $37^{\circ} \mathrm{C}$. The determined partition coefficient for $n$-butyraldehyde was 0.141 and 


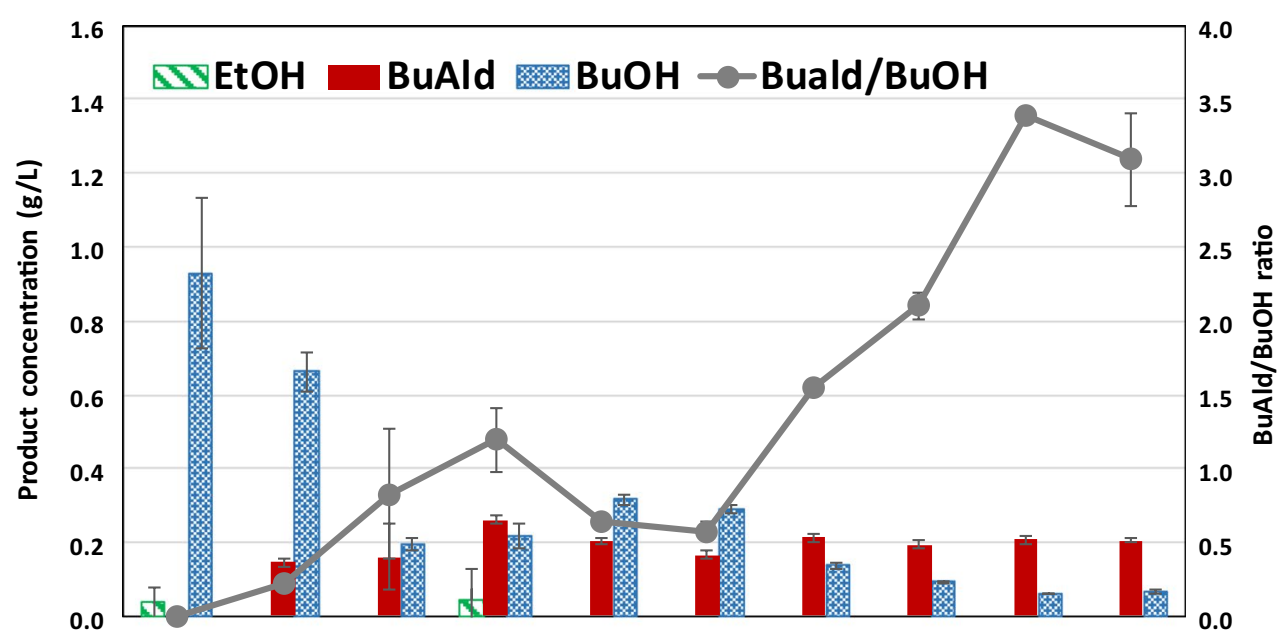

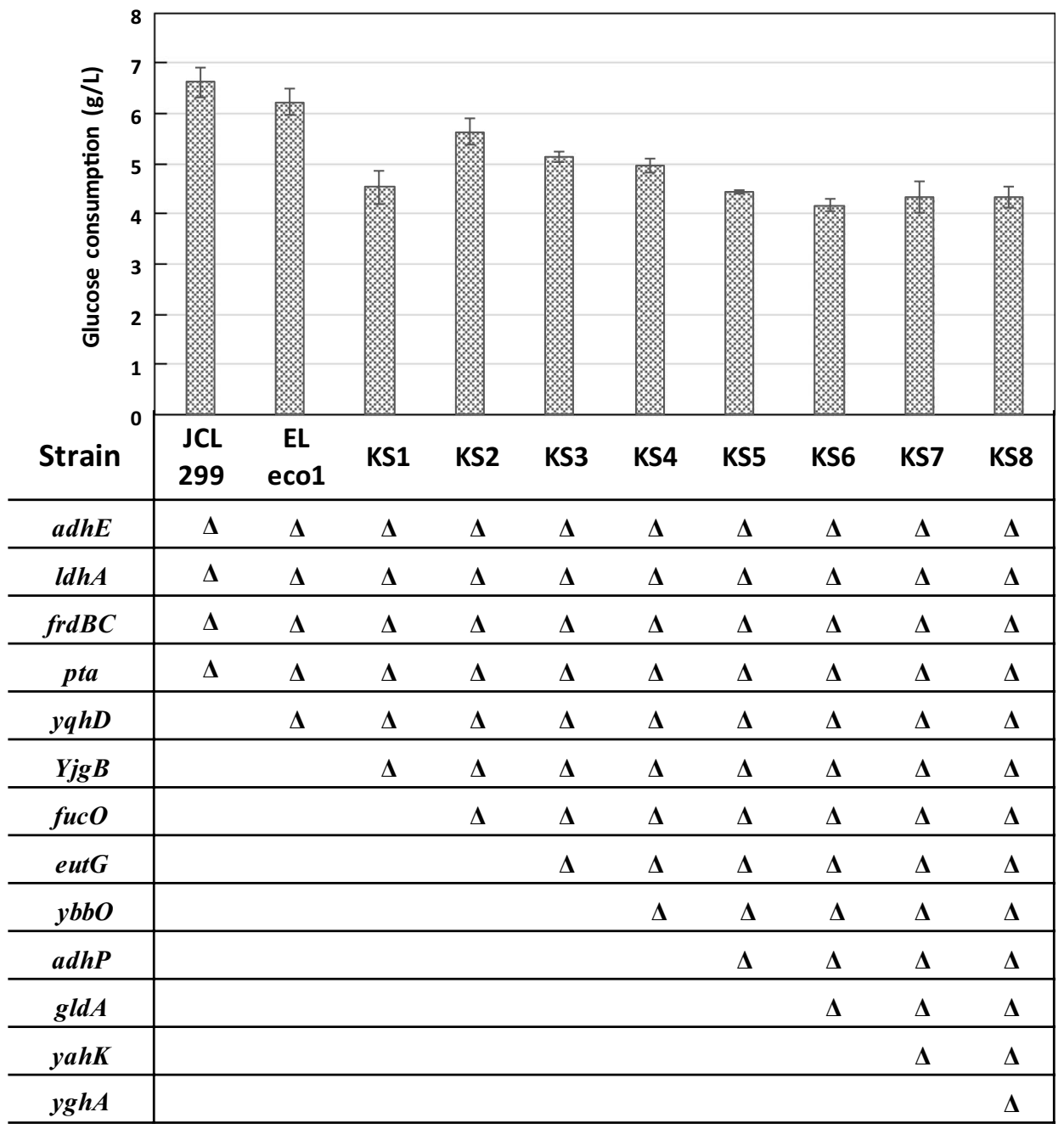

Fig. 3 -Butyraldehyde production and glucose consumption by different strains with alcohol dehydrogenase knock out in $24 \mathrm{~h}$. All strains harbor pKU48, pRW18, and pRW22 for butytaldehyde production. For complete strain and plasmid list, see Table 1. Triangles in the table indicate gene knock out. Error bars represent standard deviation of three experiments 
Table 1 Strains and plasmids

\begin{tabular}{|c|c|c|}
\hline Strain & Genotype & References \\
\hline BW25113 & $r r n B_{\mathrm{T} 14} \Delta l a c Z_{\mathrm{WJ16}} h s d \mathrm{R} 514 \triangle \operatorname{araBAD} \mathrm{AH}_{\mathrm{A} 33} \Delta r h a B A D_{\mathrm{LD} 78}$ & \\
\hline XL1-blue & recAl endA1 gyrA96 thi-1 hsdR17 supE44 relA1 lac [F' proAB lac192 $\triangle M 15 \operatorname{Tn} 10\left(\right.$ Tet $\left.\left.^{1}\right)\right]$ & Agilent Technologies \\
\hline JCL16 & BW25113/F' $\left[\right.$ traD36 proAB ${ }^{+}$laclaZ $\triangle M 15\left(\right.$ Tet $\left.\left.^{\prime}\right)\right]$ & {$[30]$} \\
\hline JCL299 & JCL16 $\triangle a d h E \triangle l d h A \triangle f r d B C \triangle p t a$ & [9] \\
\hline ELeco1 & JCL299 $\triangle y q h D$ & This study \\
\hline KS1 & $J C L 299 \triangle y q h D \Delta y j g B$ & This study \\
\hline KS2 & JCL299 $\triangle y q h D \triangle y j g B \triangle f u c O$ & This study \\
\hline KS3 & JCL299 $\triangle y q h D \triangle y j g B \Delta f u c O \triangle e u t G$ & This study \\
\hline KS4 & JCL299 $\triangle y q h D \triangle y j g B \Delta f u c O \triangle e u t G \Delta y b b O$ & This study \\
\hline KS5 & JCL299 $\triangle y q h D \Delta y j g B \Delta f u c O \Delta e u t G \Delta y b b O \triangle a d h P$ & This study \\
\hline KS6 & JCL299 $\Delta y q h D \Delta y j g B \Delta f u c O \triangle e u t G \Delta y b b O \triangle a d h P \triangle g l d A$ & This study \\
\hline KS7 & JCL299 $\Delta y q h D \Delta y j g B \Delta f u c O \triangle e u t G \Delta y b b O \Delta a d h P \Delta g l d A \Delta y a h K$ & This study \\
\hline KS8 & JCL299 $\triangle y q h D \Delta y j g B \Delta f u c O \triangle e u t G \Delta y b b O \triangle a d h P \triangle g l d A \Delta y a h K \Delta y g h A$ & This study \\
\hline Plasmid & Genotype & References \\
\hline pRW13 & $P_{\text {ack }}:$ atoB, adhE2, crt, hbd; ColE1 ori; Ampr & [19] \\
\hline pRW18 & 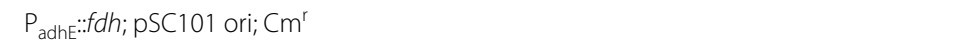 & [19] \\
\hline pRW22 & $P_{\text {adhE:::er; Cola ori; Kan }}^{r}$ & [19] \\
\hline pKU48 & $P_{\text {ack }}:$ atoB, aldh (Clostridium beijerinckii), crt, hbd; ColE1 ori; Ampr & This study \\
\hline pKU49 & $P_{\text {ack }}:$ atoB, aldh (mutant gene; Clostridium beijerinckii), crt, hbd; ColE1 ori; Ampr & This study \\
\hline pKU50 & 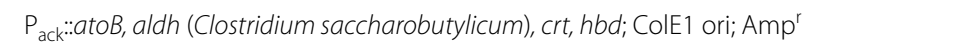 & This study \\
\hline pKU51 & $\mathrm{P}_{\text {ack: }}:$ atoB, aldh (Clostridium saccharoperbutylacetonicum N1-4), crt, hbd; ColE1 ori; Ampr & This study \\
\hline
\end{tabular}

Gene sources are as follows: atoB, acetyl-CoA acetyltransferase from E. coli; adhE2, aldehyde-alcohol dehydrogenase from Clostridium acetobutylicum; crt, crotonase from Clostridium acetobutylicum; hbd, 3-hydroxybutyryl-CoA dehydrogenase from Clostridium acetobutylicum; fdh, formate dehydrogenase from Candida boidinii, ter trans-enoyl-CoA reductase from Treponema denticola l; aldh, aldehyde dehydrogenase from sources indicated in the table

0.764 for dodecane and oleyl alcohol, respectively. This result indicated that oleyl alcohol may be a more suitable extractant for $n$-butyraldehyde as higher partition coefficient indicates the higher ratio of $n$-butyraldehyde found in the organic layer. These two extractants are only mildly toxic to $E$. coli as the growth of the $n$-butyraldehyde producing strain cultivated in the presence of either dodecane or oleyl alcohol were only slightly lowered compared to the control without any extractant (Fig. 4a), indicating the suitability of these solvents for in situ extraction. As results shown in Fig. 4 b, $n$-butyraldehyde titer significantly improved in the presence of extractant. Consistently for both dodecane and oleyl alcohol, using 1 volume of extractant outperforms that using 0.5 volume. The best condition using oleyl alcohol with a 1:1 extractant-to-culture volume ratio produced over $0.6 \mathrm{~g} / \mathrm{L}$ of $n$-butyraldehyde, representing a near three-fold improvement over no extractant. As expected, oleyl alcohol outperformed dodecane as extractant for $n$-butyraldehyde (Fig. 4c), consistent with the partition coefficients.

\section{Effect of reducing media complexity on $n$-butyraldehyde production}

Next, we evaluated the effect of yeast extract and tryptone concentration on $n$-butyraldehyde production. Using terrific broth (TB) is typically not commercially viable due to its expensive cost. Furthermore, aldehydes are reactive and can spontaneously form Schiff base with amines. Since TB contains high amounts of yeast extract and tryptone, aldehydes may likely be spontaneously reacted with the amino groups present on amino acids and oligopeptides in TB. Using M9 media with glucose as the base, we supplement 0 to $2 \%$ yeast extract or tryptone to determine an optimum level. Results are summarized in Fig. 5. Figure 5a shows the effect of yeast extract concentration on the production of $n$-butyraldehyde after $24 \mathrm{~h}$ of anaerobic incubation. $n$-Butyraldehyde titer was not significantly sensitive to yeast extract concentration between 0.125 and $2 \%$. However, if no yeast extract was added, only minimal amount of $n$-butyraldehyde was observed, indicating the importance of complex nitrogen 

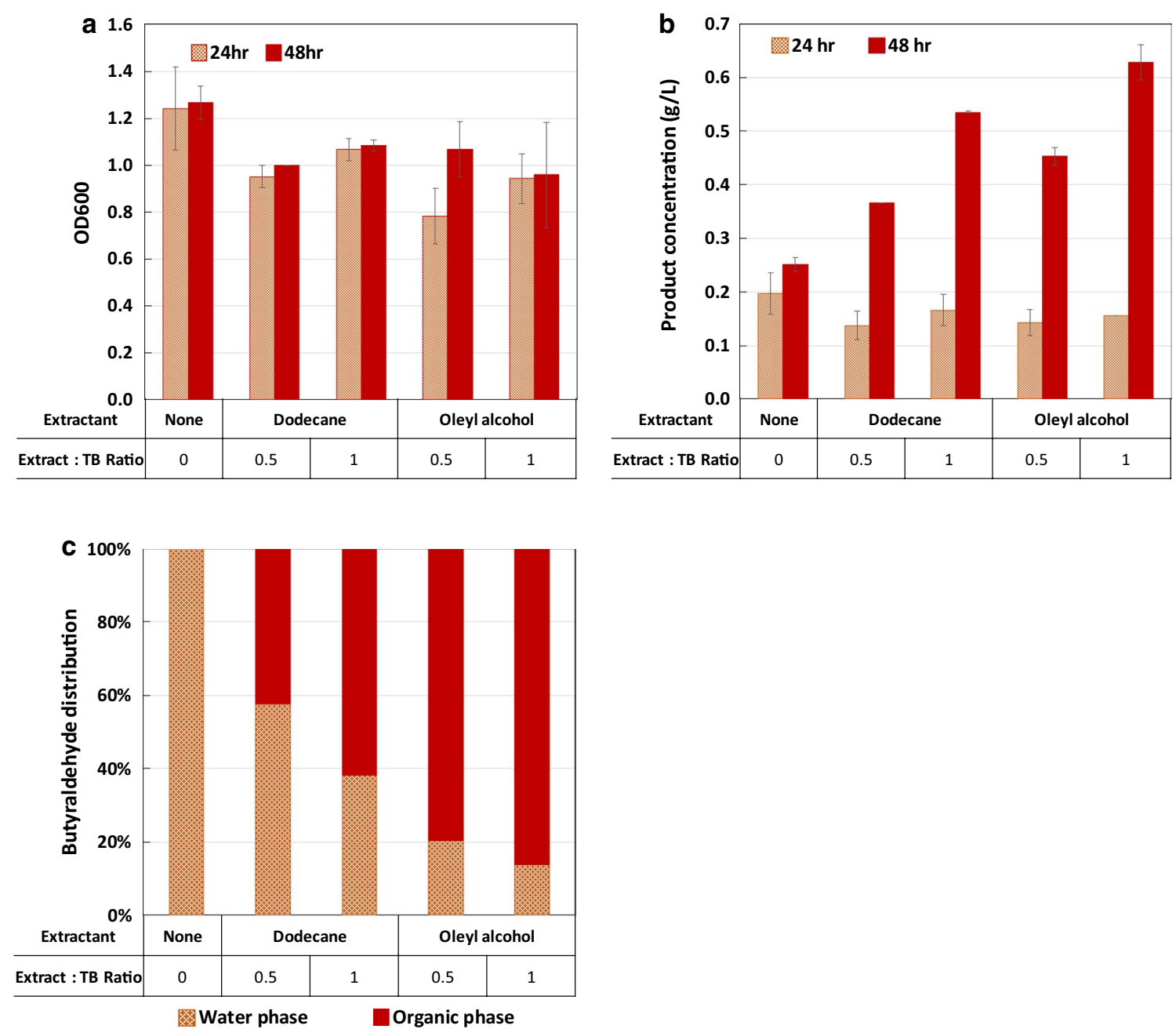

Fig. 4 n-Butyraldehyde production using two-phase extraction for in situ product removal. a Cell growth in media with extractant dodecane and oleyl alcohol overlay. b Total $n$-butyraldehyde titer using different extractants. $\mathbf{c} n$-Butyryaldehyde distribution in the culture media (water phase) and extractant (organic phase) for the 48-h samples. The ratio of extractant to TB is defined as the volume of extractant added divided by the $20 \mathrm{~mL}$ of culture (TB with $2 \%$ glucose). Error bars represent the standard deviation of triplicated experiments

source. Interestingly, those using M9 with yeast extract had a higher aldehyde-to-alcohol ratio than that using TB due to higher levels of butanol produced. 48-h postanaerobic switch (Fig. 5b) showed slight decrease in $n$-butyraldehyde and increase in $n$-butanol titer, indicating functional alcohol dehydrogenase actively converting $n$-butyraldehyde to $n$-butanol.

$n$-Butyraldehyde production was more sensitive to tryptone concentration than that of yeast extract as cultures containing 0.125 and $0.25 \%$ tryptone showed lower $n$-butyraldehyde titer compared to the corresponding concentrations of yeast extract (Fig. 5c, d). Increasing tryptone concentration led to increased $n$-butanol production, indicating that tryptone contributed towards the lowered aldehyde-to-alcohol ratio for using $\mathrm{TB}$ as production media. By comparing the components of yeast extract and tryptone from the manufacturers' manual, we noticed that tryptone has higher percentage of larger molecules with molecular weight in the range of than 500-2000 Da, indicating a larger amount of oligopeptides. On the other hand, yeast extract contains mostly smaller molecules with molecular weight less than $250 \mathrm{Da}$. It is possible that this discrepancy led to different expression patterns which may include nonspecific native alcohol dehydrogenases capable of reducing $n$-butyraldehyde. Nonetheless, the exact mechanism to why tryptone causes increase in $n$-butanol production is unclear. 

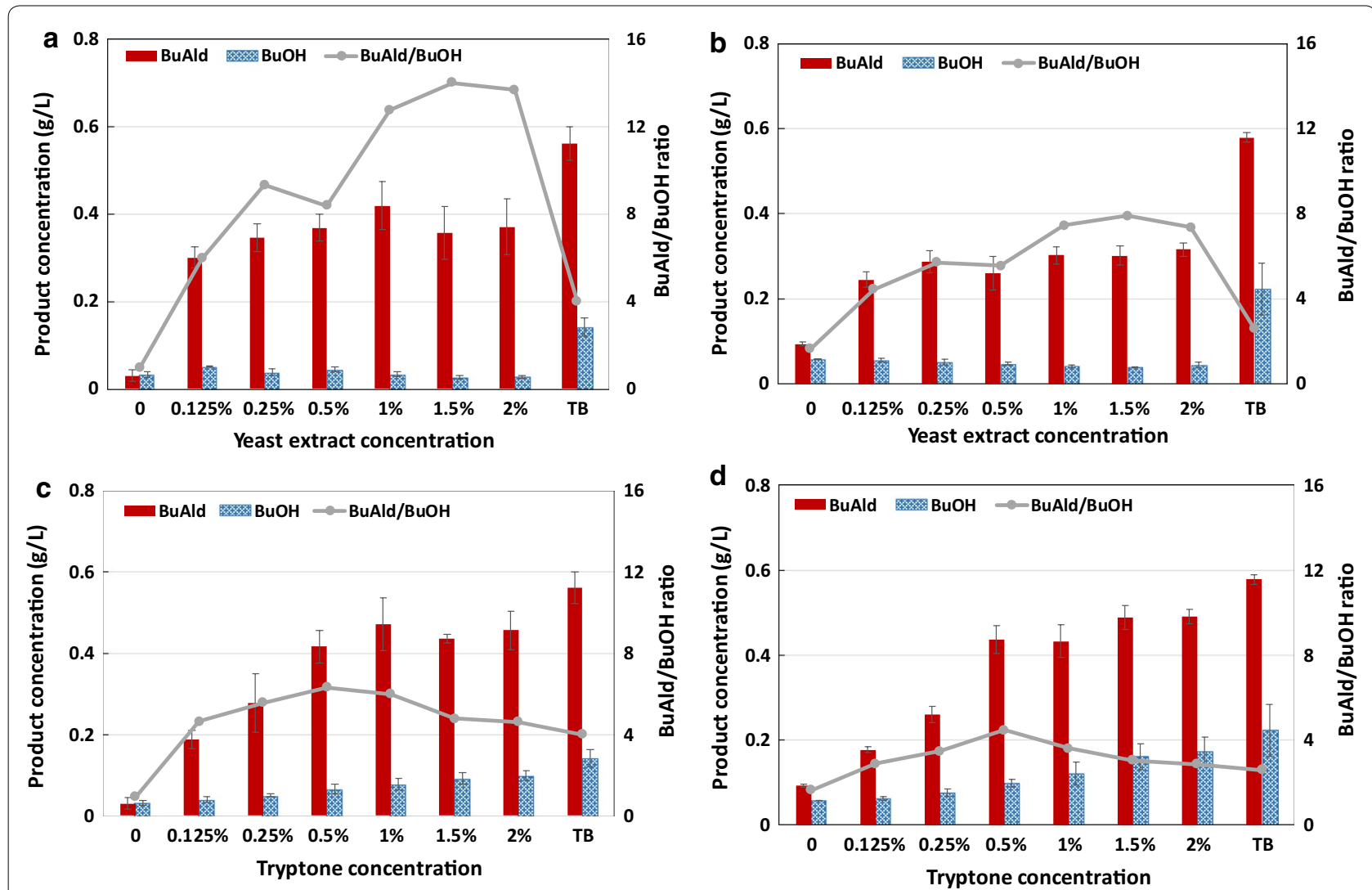

Fig. 5 Comparison of yeast extract $(\mathbf{a}, \mathbf{b})$ and tryptone $(\mathbf{c}, \mathbf{d})$ concentration in M9 glucose media for $n$-butyraldehyde production. Product concentrations and butyraldehyde-to-butanol ratios sampled at $\mathbf{a}, \mathbf{c} 24 \mathrm{~h}$ and $\mathbf{b}$, d 48-h post-switch to anaerobic condition. Strain KS8/pKU48/pRW18/ pRW22 was used for $n$-butyraldehyde production

\section{Conclusions}

This study demonstrated $n$-butyraldehyde production from glucose using engineered $E$. coli. We showed that aldh gene from $C$. beijerinckii outperformed the other aldh genes tested in achieving highest butyraldehyde-tobutanol ratio. Subsequent knockouts of endogenous $a d h$ genes including $y q h D, y j g B$, fucO, adhP, gldA, and $y a h K$, in situ product removal by oleyl alcohol, and medium optimization using M9 $2 \%$ glucose with $1-2 \%$ yeast extract significantly improved both the $n$-butyraldehyde titer (from $10 \mathrm{mg} / \mathrm{L}$ to $630 \mathrm{mg} / \mathrm{L}$ ) and butyraldehydeto-butanol ratio. Compared to E. coli glucose-based $n$-butanol production (with titer up to $15 \mathrm{~g} / \mathrm{L}$ in test tubes), $n$-butyraldehyde production using similar strain and pathway resulted in significantly lower titer. It is possible to achieve renewable $n$-butyraldehyde production via bio-butanol followed by chemical conversion. The chemical conversion of $n$-butanol to $n$-butyraldehyde is possible using $\mathrm{Cu}[27,28]$ - or Pt [29]-based catalysis. However, the $\mathrm{Cu}$-based catalysis requires high temperature of 500 to $800 \mathrm{~K}$. While the Pt-based catalysis can produce $n$-butyraldehyde from $n$-butanol at lower temperatures, leaching of the expensive Pt-based catalyst increases cost of the overall process. Therefore, sugarbased direct production of $n$-butyraldehyde remains an attractive potential direction. In order for it to become industrially viable in the future, further optimization of genetic expression, media, and product removal techniques is necessary.

\section{Additional file}

Additional file 1. Supporting information

\section{Authors' contributions}

JTK, WS, and EIL designed the experiments. JTK and WS performed the experiments. JTK, WS, and EIL analyzed the data. EIL supervised the experiments. JTK and EIL wrote the manuscript. All authors read and approved the final manuscript.

\section{Author details}

${ }^{1}$ Department of Biological Science and Technology, National Chiao Tung University, 1001 Daxue Road, Hsinchu 300, Taiwan. ${ }^{2}$ Institute of Molecular Medicine and Bioengineering, National Chiao Tung University, 1001 Daxue Road, Hsinchu 300, Taiwan. 


\section{Acknowledgements}

This work was funded by the Ministry of Science and Technology (MOST), R.O.C. Taiwan through Grant 105-2221-E-009-164, partially through MOST 106-3113-E-007-002, and the laboratory start-up fund from the National Chiao Tung University. The authors would like to thank Dr. Claire R. Shen for providing plasmids used in this study.

\section{Competing interests}

The authors declare that they have no competing interests.

\section{Availability of data and materials}

Data and material supporting the findings can be found at National Chiao Tung University Department of Biological Science and Technology in Hsinchu, Taiwan.

\section{Ethics approval and consent to participate} Not applicable.

\section{Publisher's Note}

Springer Nature remains neutral with regard to jurisdictional claims in published maps and institutional affiliations.

Received: 18 August 2017 Accepted: 26 November 2017

Published online: 04 December 2017

\section{References}

1. Raff DK. Butanals. Ullmann's Encyclopedia of Industrial Chemistry. Weinheim: Wiley; 2013

2. Kallio P, Pásztor A, Thiel K, Akhtar MK, Jones PR. An engineered pathway for the biosynthesis of renewable propane. Nat Commun. 2014;5:4731. https://doi.org/10.1038/ncomms5731.

3. Kunjapur AM, Tarasova Y, Prather KL. Synthesis and accumulation of aromatic aldehydes in an engineered strain of Escherichia coli. J Am Chem Soc. 2014;136:11644-54.

4. Rodriguez GM, Atsumi S. Isobutyraldehyde production from Escherichia coli by removing aldehyde reductase activity. Microb Cell Fact. 2012;11:90.

5. Yoon SH, Li C, Kim JE, Lee SH, Yoon JY, Choi MS, Seo WT, Yang JK, Kim JY, Kim SW. Production of vanillin by metabolically engineered Escherichia coli. Biotech Lett. 2005:27:1829-32.

6. Ni J, Tao F, Du H, Xu P. Mimicking a natural pathway for de novo biosynthesis: natural vanillin production from accessible carbon sources. Sci Rep. 2015;5:13670.

7. Rogers P, Palosaari N. Clostridium acetobutylicum mutants that produce butyraldehyde and altered quantities of solvents. Appl Environ Microbiol. 1987;53:2761-6.

8. Cho KM, Higashide W, Lee C, Rabizadeh S. Microbial production of n-butyraldehyde. Google Patents. US9777297 B2. 2014

9. Shen CR, Lan El, Dekishima Y, Baez A, Cho KM, Liao JC. Driving forces enable high-titer anaerobic 1-butanol synthesis in Escherichia coli. Appl Environ Microbiol. 2011;77:2905-15.

10. Thomason LC, Costantino N, Court DL. E. coli genome manipulation by P1 transduction. Curr Protoc Mol Biol. 2007:8:1-17.

11. Baba T, Ara T, Hasegawa M, Takai Y, Okumura Y, Baba M, Datsenko KA Tomita M, Wanner BL, Mori H. Construction of Escherichia coli K-12 inframe, single-gene knockout mutants: the Keio collection. Mol Syst Biol. 2006;2006(2):0008

12. Gibson DG, Young L, Chuang RY, Venter JC, Hutchison CA, Smith HO Enzymatic assembly of DNA molecules up to several hundred kilobases. Nat Methods. 2009;6:343-5.

13. Bond-Watts BB, Bellerose RJ, Chang MCY. Enzyme mechanism as a kinetic control element for designing synthetic biofuel pathways. Nat Chem Biol. 2011;7:222-7

14. Lan El, Liao JC. Microbial synthesis of $n$-butanol, isobutanol, and other higher alcohols from diverse resources. Bioresour Technol. 2013;135:339-49.
15. Leal NA, Havemann GD, BobikTA. PduP is a coenzyme-a-acylating propionaldehyde dehydrogenase associated with the polyhedral bodies involved in B-12-dependent 1,2-propanediol degradation by Salmonella enterica serovar Typhimurium LT2. Arch Microbiol. 2003;180:353-61.

16. Stojiljkovic I, Baumler AJ, Heffron F. Ethanolamine utilization in salmonella-typhimurium: nucleotide-sequence, protein expression, and mutational analysis of the ccha cchb eute eutj eutg euth gene-cluster. J Bacteriol. 1995;177:1357-66.

17. Lan El, Ro SY, Liao JC. Oxygen-tolerant coenzyme A-acylating aldehyde dehydrogenase facilitates efficient photosynthetic $n$-butanol biosynthesis in cyanobacteria. Energy Environ Sci. 2013;6:2672-81.

18. Toth J, Ismaiel AA, Chen JS. The ald gene, encoding a coenzyme A-acylating aldehyde dehydrogenase, distinguishes Clostridium beijerinckii and two other solvent-producing clostridia from Clostridium acetobutylicum. Appl Environ Microbiol. 1999;65:4973-80.

19. Wen RC, Shen CR. Self-regulated 1-butanol production in Escherichia coli based on the endogenous fermentative control. Biotechnol Biofuels. 2016;9:267

20. Perez JM, Arenas FA, Pradenas GA, Sandoval JM, Vasquez CC. Escherichia coli YqhD exhibits aldehyde reductase activity and protects from the harmful effect of lipid peroxidation-derived aldehydes. J Biol Chem. 2008;283:7346-53.

21. Lee C, Kim I, Lee J, Lee KL, Min B, Park C. Transcriptional activation of the aldehyde reductase YqhD by YqhC and its implication in glyoxal metabolism of Escherichia coli K-12. J Bacteriol. 2010;192:4205-14.

22. Atsumi S, Wu TY, Eckl EM, Hawkins SD, Buelter T, Liao JC. Engineering the isobutanol biosynthetic pathway in Escherichia coli by comparison of three aldehyde reductase/alcohol dehydrogenase genes. Appl Microbiol Biotechnol. 2010;85:651-7.

23. Rodriguez GM, Atsumi S. Toward aldehyde and alkane production by removing aldehyde reductase activity in Escherichia coli. Metab Eng. 2014;25:227-37.

24. Flamholz A, Noor E, Bar-Even A, Milo R. eQuilibrator-the biochemical thermodynamics calculator. Nucleic Acids Res. 2012;40:D770-5.

25. Jang HJ, Yoon SH, Ryu HK, Kim JH, Wang CL, Kim JY, Oh DK, Kim SW. Retinoid production using metabolically engineered Escherichia coli with a two-phase culture system. Microb Cell Fact. 2011;10:59.

26. George KW, Thompson MG, Kang A, Baidoo E, Wang G, Chan LJ, Adams PD, Petzold CJ, Keasling JD, Lee TS. Metabolic engineering for the highyield production of isoprenoid-based C(5) alcohols in E. coli. Sci Rep. 2015:5:11128

27. Jyothi Y, Vakati V, Satyanarayana T, Veerasomaiah P. Gas phase dehydrogenation of $n$-butanol to butyraldehyde on magnesia supported copper catalysts. Indian J Chem. 2014;53A:553-6.

28. Requies J, Güemez M, Maireles P, Iriondo A, Barrio V, Cambra J, Arias P. Zirconia supported Cu systems as catalysts for $n$-butanol conversion to butyraldehyde. Appl Catal A. 2012;423:185-91.

29. Gandarias I, Nowicka E, May BJ, Alghareed S, Armstrong RD, Miedziak PJ, Taylor SH. The selective oxidation of $n$-butanol to butyraldehyde by oxygen using stable Pt-based nanoparticulate catalysts: an efficient route for upgrading aqueous biobutanol. Catal Sci Technol. 2016;6:4201-9.

30. Atsumi S, Cann AF, Connor MR, Shen CR, Smith KM, Brynildsen MP, Chou KJY, Hanai T, Liao JC. Metabolic engineering of Escherichia coli for 1-butanol production. Metab Eng. 2008:10:305-11.

\section{Submit your next manuscript to BioMed Central} and we will help you at every step:

- We accept pre-submission inquiries

- Our selector tool helps you to find the most relevant journal

- We provide round the clock customer support

- Convenient online submission

- Thorough peer review

- Inclusion in PubMed and all major indexing services

- Maximum visibility for your research

Submit your manuscript at www.biomedcentral com/submit 